\title{
A CLASS OF GRAPHS
}

\author{
BY \\ PAUL KELLY AND DAVID MERRIELL
}

We consider even order graphs in which no two points have more than one join and no point is joined to itself. In such a graph $G$, of order $2 n,[A, B]$ denotes an "equipartition of $G$ " if $A$ and $B$ are subgraphs of $G$ of order $n$ whose vertex sets are disjoint. When $A$ is isomorphic to $B$ the equipartition is a "bisection of $G$." We establish that for every integer $n>1$ there exists a nontrivial graph of order $2 n$ which "has all bisections," that is, a graph such that every equipartition is a bisection. (In this connection, a graph is trivial if it has all joins or no joins.) The class of graphs having all bisections is completely characterized.

TheOREM 1. An even order graph $G$ is regular if and only if, for every equipartition $[A, B] A$ and $B$ have the same number of joins.

Proof. Let the vertex $p_{i}$ have degree $k_{i}, i=1,2, \cdots, 2 n$, with the points ordered so that $k_{1} \leqq k_{2} \leqq \cdots \leqq k_{2 n}$. Place points $p_{1}$ to $p_{n}$ in $A$ and the remaining points in $B$. Let $x_{i}$ denote the degree of $p_{i}$ in the half graph $A$ or $B$ to which it belongs, and set $y_{i}=k_{i}-x_{i}$. Since the number of joins in a graph is half the sum of its degrees, $\sum_{i=1}^{n} x_{i}=\sum_{i=n+1}^{2 n} x_{i}$, and since the number of joins from $A$ to $B$ is the same as from $B$ to $A, \sum_{i=1}^{n} y_{i}=\sum_{i=n+1}^{2 n} y_{i}$. Thus, $\sum_{i=1}^{n}\left(x_{i}+y_{i}\right)=\sum_{i=n+1}^{2 n}\left(x_{i}+y_{i}\right)$, or $\sum_{i=1}^{n} k_{i}=\sum_{i=n+1}^{2 n} k_{i}$, which implies $k_{1}=k_{2}=\cdots=k_{2 n}$.

Conversely, let $G$ be regular of degree $k$ and suppose that there is an equipartition in which $A$ has fewer joins than $B$. Then, by the same reasoning as before, $\sum_{i=1}^{n} x_{i}<\sum_{i=n+1}^{2 n} x_{i}$, while $\sum_{i=1}^{n} y_{i}=\sum_{i=n+1}^{2 n} y_{i}$, so $\sum_{i=1}^{n}\left(x_{i}+y_{i}\right)$ $<\sum_{i=n+1}^{2 n}\left(x_{i}+y_{i}\right)$, which is impossible, since $x_{i}+y_{i}=k$ for all $i$.

COROLlary. A graph which has all bisections is regular.

If $T(G)$ is the transpose of $G$ (or complement), obtained by reversing all the join relationships in $G$, an equipartition of $G$ is also an equipartition of $T(G)$ and both are bisections or neither is. Since it is easily verified that a graph has all bisections if it consists solely of components which are sticks, we have:

THeOREM 2. A regular graph of order $2 n$ has all bisections if its degree is 1 or $2 n-2$.

THEOREM 3. If a regular graph $G$, of order $2 n$ and degree $k>1$, has all bi-

Presented to the Society, January 27, 1960, under the title $A$ special class of graphs; received by the editors November 16,1959 . 
sections and is not connected, then it consists of two components; these are either two complete graphs of order $n$ or else are two four-circuits.

Proof. Let $G_{1}, G_{2}, \cdots, G_{h}$ denote the components of $G$ with the corresponding orders $r_{1} \geqq r_{2} \geqq \cdots \geqq r_{h} \geqq k+1$. Then $r_{1}$ is not less than $n$. For $0<n-r_{1} \leqq h-1$ would imply an equipartition $[A, B]$ in which $A$ consisted of $G_{1}$ together with single points chosen from $n-r_{1}$ of the remaining components. Because $k>1, B$ could have no isolated points and $[A, B]$ would not be a bisection. On the other hand, for $0<n-r_{1}$ and $n-r_{1}>h-1$ there would exist $[A, B]$ in which $A$ consisted of $G_{1}$ and at least one point from each of the other components. Then $B$ would have no component of order $r_{1}$ and $[A, B]$ would not be a bisection. Now since a connected graph has connected subgraphs of every lower order, and since $r_{1} \geqq n$, there exists a bisection $[A, B]$ in which $A$ is an $n$ th-order, connected subgraph of $G_{1}$. Hence $B$ is connected, which implies that $h=2$ and that $r_{1}=r_{2}=n$.

Next suppose that the isomorphic components $G_{1}$ and $G_{2}$ are not complete graphs. There are then two points, $p$ and $q$, in $G_{1}$ which are not joined. Let $B_{1}$ be the subgraph of $G_{1}$ defined on the remaining vertices, and in the bisection $[A, B]$ take $B$ to consist of $B_{1}$ together with any two points of $G_{2}$ which are joined. If $k>2$, a contradiction is reached by the fact that $p$ and $q$ are isolated in $A$ while every point of $B$ has degree at least 1 . If $k=2$, then $G_{1}$ and $G_{2}$ are $n$-circuits and, for $n \neq 4, B$ has at most one isolated point. That two disjoint four-circuits form a graph with all bisections is easily checked.

Theorem 4. Let $P$ and $Q$ denote complete graphs of order $n$. A graph $G$ is defined in one of the following ways: (I) it consists of two components $P$ and $Q$; (II) it consists of $P$ and $Q$ and $n$ additional joins such that each point of $P$ joins one point of $Q$ and conversely; (III) it is the transpose of a graph defined by (I) or (II). Then $G$ has all bisections.

Proof. The conclusion in the case of (I) follows trivially from the properties that subgraphs of complete graphs are complete, and complete graphs of the same order are isomorphic. To establish method (II), let

$$
P=\left\{p_{1}, p_{2}, \cdots, p_{n}\right\}, \quad Q=\left\{q_{1}, q_{2}, \cdots, q_{n}\right\},
$$

with $p_{i}$ joined to $q_{i}, i=1,2, \cdots, n$. In an arbitrary equipartition $[A, B]$, $A$ possesses $s$ points of $P$ whose corresponding join-points in $Q$ are not in $A$ and $t$ points of $P$ whose corresponding join-points in $Q$ are in $A$. Then, without loss of generality, we may suppose that

$$
A=\left\{p_{1}, p_{2}, \cdots, p_{s}, p_{s+1}, p_{s+2}, \cdots, p_{s+t}, q_{8+1}, q_{8+2}, \cdots, q_{s+t}\right\}
$$

and

$$
B=\left\{q_{1}, q_{2}, \cdots, q_{s}, q_{s+t+1}, p_{s+t+2}, \cdots, p_{s+2 t}, q_{s+t+1}, q_{s+t+2}, \cdots, q_{s+2 t}\right\},
$$

where $s+2 t=n$. Since the correspondence 


$$
\left.p_{i} \rightarrow q_{i}, i=1,2, \cdots, s \quad \begin{array}{rl}
p_{s+i} & \rightarrow q_{s+t+i} \\
q_{s+i} & \rightarrow p_{s+t+i}
\end{array}\right\}, \quad i=1,2, \cdots, t,
$$

is easily seen to be join preserving, $[A, B]$ is a bisection. Method (III) follows from the properties of transposition.

We next wish to establish that every nontrivial graph $G$ which has all bisections, and which is not two disjoint four-circuits or its transpose, is constructable by the methods of Theorem 4 . To this end we introduce the subgraphs of $G, J(p)$ and $N(p)$, corresponding to each point $p$ of $G$. The first, $J(p)$, consists of all points joined to $p$ in $G$, the second, $N(p)$, of all points not joined in $G$ to any point of $p \cup J(p)$. Relative to these subgraphs, we establish the following lemmas.

Lemma 1. If $G$, of order $2 n$ and degree $k \leqq n-1$, has all bisections and is connected, then $J(p) \neq J(q)$ for any pair of vertices $p, q$.

Proof. Assume that $J(p)=J(q)$. Construct an equipartition $[A, B]$ with $p \in A$ and $B_{1}=q \cup J(p) \subset B$. Complete $B$, if necessary, by successively assigning to it points which either join a point of $B_{1}$ or else join a point previously assigned to $B$. Since $p$ is isolated in $A$ under any completion of $A$, and the construction of $B$ yields no isolated point in $B$, the construction cannot complete $B$, which contradicts the connectedness of $G$.

Lemma 2. If $G$, of order $2 n$ and degree $k \leqq n-1$, is connected and has all bisections, then for each $p$ in $G$ the order of $N(p)$ is $n-k$.

Proof. The connectedness of $G$, with $n \geqq k+1$, implies $k>1$. Let $p$ be any vertex of $G$ and consider any bisection $[A, B]$ such that $p \cup J(p) \subset A$. Because $A$ and $B$ are isomorphic, there exists a point $b_{1}$ of degree $k$ in $B$. Since $b_{1} \in N(p)$, if $k=n-1$ the order of $N(p)$ is at least $n-k$. For $k<n-1$, let $a_{1}, a_{2}, \cdots, a_{n-k-1}$ denote the points of $A$ not in $p \cup J(p)$. We now modify $[A, B]$ to a new bisection $\left[A_{1}, B_{1}\right]$ by interchanging $a_{1}$ and $b_{1}$. Then $B_{1}$ has a point $b_{2} \neq b_{1}$ of degree $k$ in $B_{1}$ corresponding to $p$ in $A_{1}$. Next, modify $\left[A_{1}, B_{1}\right]$ to $\left[A_{2}, B_{2}\right]$ by interchanging $a_{2}$ and $b_{2}$. Then $B_{2}$ has a point $b_{3}$ of degree $k$ in $B_{2}$. Continuing this process, until $a_{n-k-1}$ is exchanged for $b_{n-k-1}$, we obtain $n-k$ distinct points $b_{1}, b_{2}, \cdots, b_{n-k}$ which must belong to $N(p)$ since each is of degree $k$ in some half-graph disjoint from $p \cup J(p)$. Thus the order of $N(p)$ is at least $n-k$.

Now we form an equipartition $\left[A^{\prime}, B^{\prime}\right]$ with $p \in A^{\prime}$ and $J(p) \subset B^{\prime}$, and assign to $A^{\prime}$ as many points of $N(p)$ as possible. Suppose that $A^{\prime}$ can be completed with $n-1$ points from $N(p)$ forming the subgraph $N_{1}(p)$ in $A^{\prime}$. Corresponding to $p$ in $A^{\prime}$ there is a point $q_{1}$ which is isolated in $B^{\prime}$. Because $k>1$ and $J\left(q_{1}\right) \subset A^{\prime}, q_{1}$ is not in $J(p)$ but in the remainder set of $B^{\prime}$, say $R_{1}$, and therefore $J\left(q_{1}\right) \subset N_{1}(p)$. In $G$ there is some path from $q_{1}$ to $p$. This path must first join $q_{1}$ to a point of $J\left(q_{1}\right) \subset N_{1}(p)$, and since no point of $N_{1}(p)$ joins 
any point of $p \cup J(p)$, the path must ultimately return to $R_{1}$ from some point $p_{1} \in N_{1}(p)$ which is joined to a point $\bar{q}_{1} \in R_{1}$, and $\bar{q}_{1} \neq q_{1}$. Now modify the bisection to one $\left[A_{1}^{\prime}, B_{1}^{\prime}\right]$ by interchanging $q_{1}$ and $\bar{p}_{1}$ in $\left[A^{\prime}, B^{\prime}\right]$. Because $q_{1} \in N(p)$, the points of $A_{1}^{\prime}$ other than $p$ again form a subgraph $N_{2}(p)$ of $N(p)$ so the isolated point $q_{2}$ of $B_{1}^{\prime}$ is not in $J(p)$ but in the remainder of $B_{1}^{\prime}$, say $R_{2}$. Also $q_{2}$ is not $\bar{p}_{1}$ or $\bar{q}_{1}$ since these are joined in $B_{1}^{\prime}$. Again, a path in $G$ from $q_{2}$ to $p$ must cross to $N_{2}(p)$ and ultimately return to $B_{1}^{\prime}$ via a point $\bar{p}_{2}$ of $N_{2}(p)$ which is joined in $G$ to a point $\bar{q}_{2}$ of $R_{2}$, and $\bar{q}_{2} \neq q_{2}$. We then exchange $p_{2}$ and $q_{2}$ in $\left[A_{1}^{\prime}, B_{1}^{\prime}\right]$ to form $\left[A_{2}^{\prime}, B_{2}^{\prime}\right]$. None of the points $\bar{p}_{1}, \bar{q}_{1}$, $\bar{p}_{2}, \bar{q}_{2}$ is isolated in $B_{2}^{\prime}$ so there is a distinct isolated point $q_{3}$ and as before it cannot belong to $J(p)$. Continuing, in less than $n-k$ of these interchanges all of the original points of $R_{1}$ can be replaced by points which have degree at least one in the $B$-half-graph. Thus an equipartition $\left[A_{i}^{\prime}, B_{i}^{\prime}\right]$ can be constructed such that $p$ is isolated in $A_{i}^{\prime}$ and no point is isolated in $B_{i}^{\prime}$.

The above contradiction shows that the order of $N(p)$ must be less than $n-1$, hence that $p \cup N(p)$ is a proper subset of $A^{\prime}$. Since every point of $B^{\prime}$ not in $J(p)$ joins some point in $J(p)$, the isolated point $q$ of $B^{\prime}$ corresponding to $p$ is in $J(p)$. Then $J(q) \subset A^{\prime}$. Because $J(q) \cap N(p)$ is null, this shows that the order of $N(p)$ is at most $n-k$. This, with the previous result that the order is at least $n-k$, shows the order is $n-k$.

THEOREM 5. Let $G$ be a graph with all bisections whose order is $2 n$ and whose degree is $k$, where $1<k<2 n-1$. If $G$ is not two disjoint four-circuits or its transpose, then $k$ is $n$ or $n-1$ and $G$ can be constructed by the methods of Theorem 4.

Proof. Let $G^{\prime}$ denote that one of the graphs $G$ and $T(G)$ whose degree $k^{\prime}$ is less than $n$. If $G^{\prime}$ is disconnected, then by Theorem 3 its degree is $n-1$ and it can be constructed by (I) of Theorem 4, while $T\left(G^{\prime}\right)$ has degree $n$ and can be constructed by (III) of Theorem 4 .

Next suppose $G^{\prime}$ is connected. For an arbitrary point $p$ consider the set $C$ of all the bisections $[A, B]$ of $G^{\prime}$ such that $p \cup N(p) \subset A$ and $J(p) \subset B$. The isolated point in $B$, corresponding to $p$ in $A$, must belong to $J(p)$ for otherwise it would be in $N(p)$ and hence in $A$. Moreover, for each partition in $C$, $J(p)$ has exactly one point isolated in $B$. For if there were two, say $q_{1}$ and $q_{2}$, $J\left(q_{1}\right)$ and $J\left(q_{2}\right)$ would both consist of the $k^{\prime}$ points of $A$ not in $N(p)$, contradicting Lemma 1. Also since $A$ consists of $p \cup N(p) \cup J(q)$, where $q$ is the point of $J(p)$ isolated in $B$, different bisections in $C$ have different points isolated in $B$. Therefore $C$ has at most $k^{\prime}$ bisections. On the other hand, $A$ has $k^{\prime}-1$ unspecified points out of $n-1$ points not fixed in either $A$ or $B$, so there are

$$
\left(\begin{array}{l}
n-1 \\
k^{\prime}-1
\end{array}\right)
$$


elements in $C$. For $k^{\prime}<n-1$,

$$
\left(\begin{array}{l}
n-1 \\
k^{\prime}-1
\end{array}\right)>n-1>k^{\prime}
$$

which is contradictory. Thus $k^{\prime}=n-1$, so there are

$$
\left(\begin{array}{l}
n-1 \\
k^{\prime}-1
\end{array}\right)=n-1
$$

elements in $C$ and hence $n-1$ isolated points in $J(p)$. The bisection $\left[A_{1}, B_{1}\right]$, where $A_{1}=J(p) \cup N(p)$ consists of two empty half graphs. Therefore $T\left(G^{\prime}\right)$, of degree $n$, can be constructed by method (II) of Theorem 4 , and $G^{\prime}$ by method (III). Since $G$ is either $G^{\prime}$ or $T\left(G^{\prime}\right)$, the theorem follows.

With the construction known for every graph $G$ having all bisections, it is easy to verify the following properties of $G$ : for each bisection $[A, B]$ there is an involutory automorphism of $G$ which interchanges $A$ and $B$; for $p, q$ in $G, p \cup J(p)$ and $q \cup J(q)$ are isomorphic; all the subgraphs of order $2 n-1$ are isomorphic to each other.

University of CALIFornia,

Santa Barbara, California 